\title{
Measurement of Composition and Strain by STEM
}

\author{
A. Rosenauer, ${ }^{1}$ K. Müller, ${ }^{1}$ T. Mehrtens, ${ }^{1}$ M. Schowalter, ${ }^{1}$ J. Zweck, ${ }^{2}$ R. Fritz, ${ }^{3}$ K. Volz ${ }^{3}$ \\ ${ }^{1}$ Institute of Solid State Physics, University of Bremen, Otto-Hahn-Allee 1, 28359 Bremen, Germany \\ ${ }^{2}$ Institute of Experimental and Applied Physics, University of Regensburg, Universitätsstraße \\ 31, 93040 Regensburg, Germany \\ ${ }^{3}$ Materials Science Center and Faculty of Physics, Philipps Universität Marburg, \\ Hans-Meerwein-Straße, 35032 Marburg, Germany
}

In this contribution we demonstrate methods for measurement of composition and strain by scanning transmission electron microscopy (STEM). On the one hand, STEM combined with a high angle annular dark field detector allows Z-contrast imaging with high material contrast that can be used for quantification of composition. The suggested method is based on comparison of experiment with image simulation [1], facilitated by normalizing the intensity with respect to the incident electron beam $[2,1]$. As an example, Fig. 1a shows a part of a high-resolution STEM image of an InGaN quantum well buried in GaN. First, we apply a Wiener filter for noise reduction, being followed by detecting the positions of the atomic columns (Fig. 1b). Then the original image is segmented into Voronoi cells (Fig. 1c), for which we compute the mean normalized intensity (Fig. 1d). Comparison with image simulations yields the local specimen thickness (Fig. 1e) and the indium concentration for each atomic column (Fig. 1f). Image simulations were performed with the STEMsim program [3] in the frozen lattice approximation and taking into account static atomic displacements (SAD), which occur due to the different covalent radii of In and Ga atoms. For the calculation of SADs we used the LAMMPS code [4] and Stillinger-Weber empirical potentials with parameters published by Lei et al. [5]. As a critical issue we also address a possible structural degradation of InGaN layers during illumination with electrons. As an example, Smeeton et al. [6] observed a clustering of indium after a few minutes irradiation time if parallel beam illumination was applied. In contrast, we could not detect any significant change of image contrast or composition during acquisition of 32 images with $80 \mathrm{~s}$ exposure time corresponding to a total irradiation time of 42 minutes. The high stability of the InGaN layers can be explained by the significantly smaller electron dose in our STEM experiments compared to that given in reference [6].

Acquisition of a series of several hundreds of diffraction patterns along a STEM line scan, on the other hand, can be used for evaluation of strain profiles. We tested three different algorithms in the field of (circular) pattern recognition that are able to detect diffracted disc positions accurately, from which the strain in growth direction is calculated. Although the three approaches are very different as one is based on edge detection, one on rotational averages and one on cross-correlation with masks, it is found that identical strain profiles (Fig. 2b) result for the $\operatorname{In}_{\mathrm{x}} \mathrm{Ga}_{1-\mathrm{x}} \mathrm{N}_{\mathrm{y}} \mathrm{As} \mathrm{s}_{-\mathrm{y}} / \mathrm{GaAs}$ heterostructure shown in Fig. 2(a) consisting of five compressively and tensile strained layers. We achieve a precision of strain measurements of 7-9 $\cdot 10_{-4}$ and a spatial resolution of $0.5-0.7 \mathrm{~nm}$ over the whole width of the layer stack which was $350 \mathrm{~nm}$.

\section{References}

[1] A. Rosenauer, et al., Ultramicroscopy 109 (2009) 1171.

[2] J.M. LeBeau and S. Stemmer, Ultramicroscopy 108 (2008) 1653.

[3] A. Rosenauer and M. Schowalter, Springer Proceedings in Physics 120 (2007) 169.

[4] S. J. Plimpton, J. Comp. Phys. 117 (1995), p. 1. 
[5] H.P. Lei, et al., Superlattices and Microstructures 40 (2006) 464.

[6] T.M. Smeeton, et al., Appl. Phys. Lett. 83 (2003) 5419.

[7] This research was supported by the Deutsche Forschungsgesellschaft under contract numbers RO2057/8-1, SCHO1196/3, V0805/4 and V0805/5.

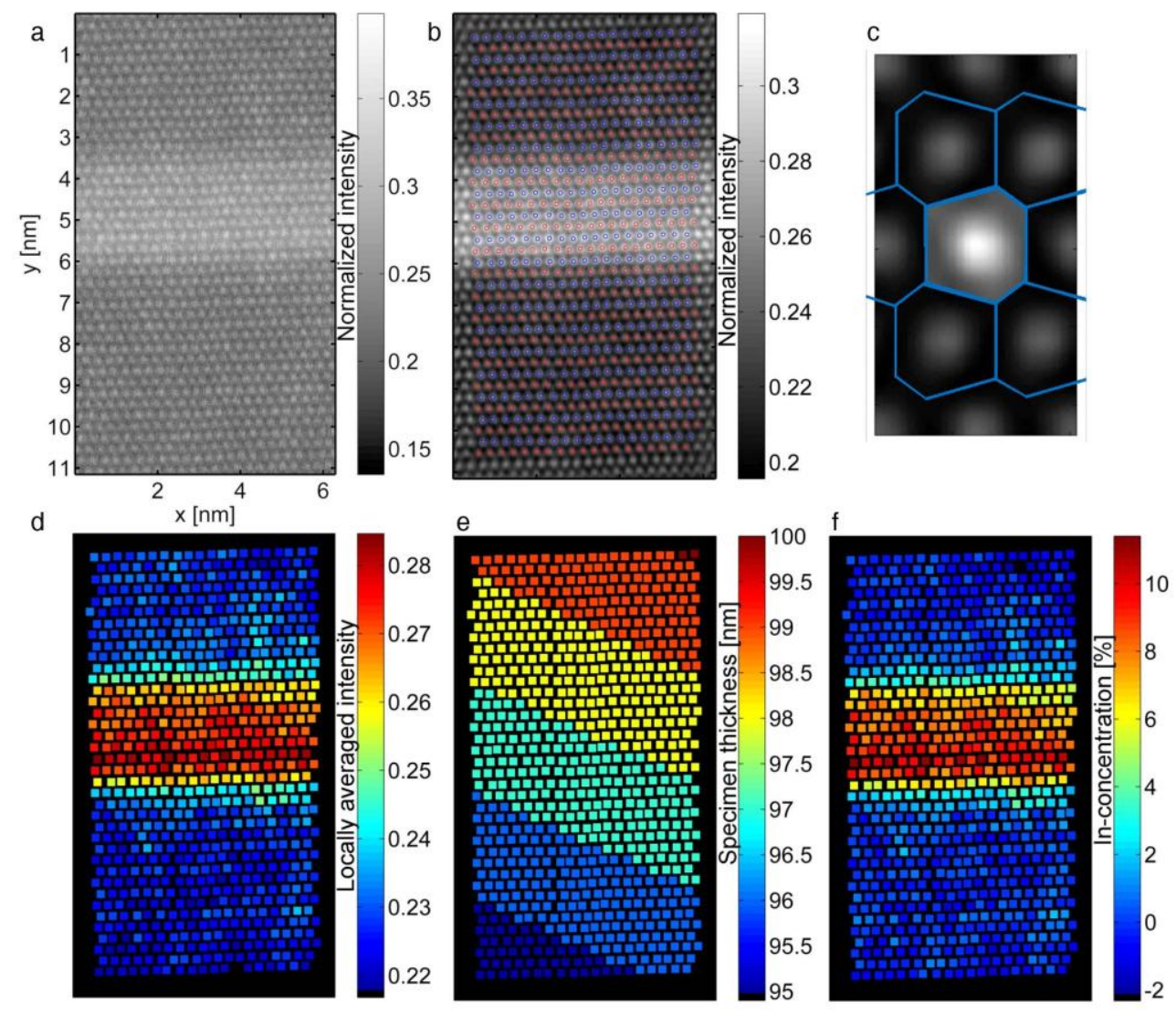

FIG. 1. (a) Small part of a STEM image, (b) noise filtered image and detected atom column positions marked with red and blue circles, (c) segmentation of the image, (d)-(f) color coded maps of (d) locally averaged image intensity, (e) evaluated specimen thickness and (f) evaluated In-concentration.
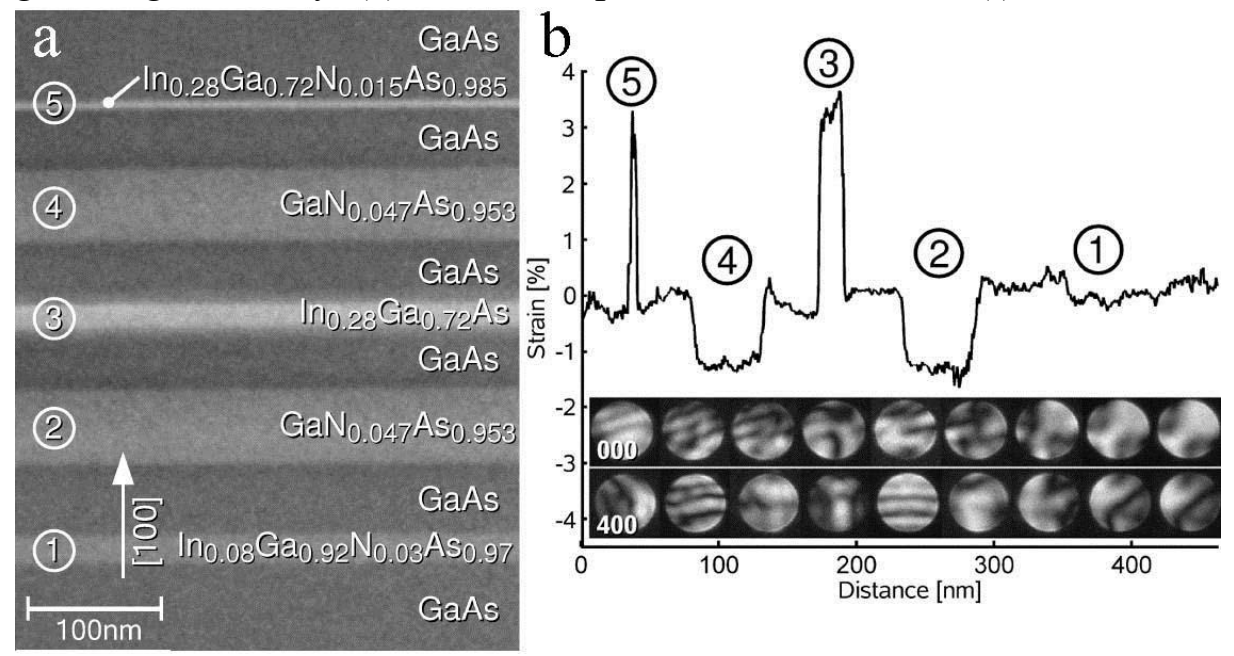

FIG. 2. (a) HAADF STEM image of a sequence of strained layers pseudomorphically grown on GaAs. (b) Strain profile measured along a line scan and diffraction discs for some positions on the line scan. 